\title{
Primary Vitreoretinal Lymphoma Masquerading as Refractory Retinitis
}

\author{
Ofira Zloto Amir E. Abd Elkader Ido Didi Fabian \\ Vicktoria Vishnevskia-Dai
}

The Goldschleger Eye Institute, Sheba Medical Center, Tel Hashomer, and The Sackler

Faculty of Medicine, Tel Aviv University, Tel Aviv, Israel

\section{Key Words}

Primary vitreoretinal lymphoma $\cdot$ Primary central nervous system lymphoma $\cdot$ Posterior uveitis · Diagnostic vitrectomy · Intravitreal methotrexate

\begin{abstract}
Purpose: To report a case of a patient with primary vitreoretinal lymphoma masquerading as retinitis. Methods: Retrospective review of the patient's clinical, histopathological and imaging records. Results: Cytopathology was negative for malignancy, and preliminary polymerase chain reaction results supported the diagnosis of varicella zoster virus retinitis. Therefore, the patient was treated with antiviral therapy. However, under this treatment, the retinitis progressed. As a result, primary vitreoretinal lymphoma was suspected, and empirical treatment with intravitreal methotrexate injections was started. Under this treatment, the ocular features improved. Five months after initial ocular presentation and ocular resolution, the patient presented with central nervous system lymphoma. Conclusion: This case should raise the awareness of the variable clinical presentations, the challenging diagnosis and treatment of primary vitreoretinal lymphoma. All cases should be continuously systemically evaluated.

(C) 2015 The Author(s)

Published by S. Karger AG, Basel
\end{abstract}

\section{Introduction}

Primary vitreoretinal lymphoma (PVRL) is a subset of primary central nervous system (CNS) lymphoma, which in most of the cases is a form of non-Hodgkin's lymphoma of diffuse large B-cell type [1]. Symptoms at presentation include blurred vision and/or floaters, but visual acuity (VA) can be preserved [2]. On examination, there is usually no involvement of the anterior segment. However, diffuse vitreous opacities, retinal lesions with subretinal and

KARGER 125/s $\quad \begin{aligned} & \text { Vicktoria Vishnevskia-Dai, MD } \\ & \text { Department of Ophthalmology } \\ & \text { The Goldschleger Eye Institute, Sheba Medical Center } \\ & \text { Tel Hashomer 52621 (Israel) } \\ & \text { E-Mail vivida65@gmail.com }\end{aligned}$


Zloto et al.: Primary Vitreoretinal Lymphoma Masquerading as Refractory Retinitis

subretinal pigment epithelium infiltrates can be seen [3]. The diagnosis of PVRL is challenging because it often mimics posterior uveitis. As posterior uveitis, initially, it can be responsive to corticosteroid treatment, but later, as opposed to posterior uveitis, the disease can be refractory to steroid treatment [4]. Vitreous biopsy is the gold standard for diagnosis of the disease [5]. Once the diagnosis is confirmed, if there is no CNS involvement, the treatment of choice includes intravitreal injections of methotrexate or rituximab [6]. However, the prognosis is poor, and 42-92\% will develop primary CNS lymphoma within 8-29 months [7]. We report a patient with PVRL who presented with refractory retinitis. He was treated empirically with serial intravitreal methotrexate injections and responded well to treatment without established diagnosis. Five months after the initial presentation (3 months after ocular improvement), CNS lymphoma was diagnosed.

\section{Case Report}

A 64-year-old male was examined in our institution due to floaters in his right eye. His medical history included controlled hypertension treated with losartan potassium. There was no ocular history. The best Snellen VA at presentation was 20/32 in the right eye and $20 / 20$ in the left eye. Slit-lamp examination of the right eye revealed normal anterior segment, cells in the vitreous and a white-yellow lesion with few retinal and subretinal hemorrhages in the inferior retina, without macular or optic disc involvement (fig. 1a). B-scan ultrasonography of the right eye displayed vitreous opacities and inferior retinal thickening with no involvement of the choroid layer. He was hospitalized with suspicion of retinitis. His work-up examinations included blood test (blood count, biochemistry profile, inflammation profile, collagenogram, viral serology), urine test, Mantoux test, lumbar puncture (LP) and anterior chamber tap (AC Tap). All of the blood tests except for the inflammation profile were normal. Urine test, Mantoux test and LP were normal. AC Tap for polymerase chain reaction (PCR) was positive for varicella zoster virus (VZV) and negative for herpes simplex virus 1 and 2 (HSV-1 and HSV-2), cytomegalovirus (CMV) and tuberculosis (TB). AC Tap cytology was negative. Due to severe claustrophobia, the patient underwent computed tomography of the orbit and brain, which was normal, but not magnetic resonance imaging (MRI).

In accordance with the clinical presentation and the AC Tap results, the working diagnosis at that time was viral retinitis, and he was treated with intravenous acyclovir and oral prednisone. Two weeks later, his VA was stable. However, at examination of the right eye, the inferior lesion had progressed to the inferior border of the optic disc and involved the macula but not the fovea (fig. 1b). Optical coherence tomography (OCT) examination demonstrated subretinal fluid, and the macular center thickness (MCT) was measured 614 $\mu \mathrm{m}$ (fig. 2a). Now, the patient agreed to undergo brain and orbit MRI scan, which was normal (fig. 3a). In light of the progression of the disease, 4 intravitreal injections of Foscarnet (twice weekly) and a single Kenalog injection were added. Moreover, a second AC Tap was performed, and at this time, as opposed to the first time, the PCR for VZV was negative along with negative cytology and negative HSV-1, HSV-2, CMV, toxoplasma and TB results. Therefore, sutureless combined 25-gauge diagnostic vitrectomy was performed. PCR tests for bacteria and viruses were performed. The specimens were prepared as cell block paraffin and stained with hematoxylin and eosin, Gram and periodic acid-Schiff stains. Furthermore, immunohistochemical stainings for CD3, CD20 and CD79a were performed. Cell block paraffin of the vitreous disclosed few normal lymphocytes. In the cell blocks, the cells were strongly 
Zloto et al.: Primary Vitreoretinal Lymphoma Masquerading as Refractory Retinitis

immunoreactive for CD3 (fig. 1d) but not for CD20. The cytopathologic diagnosis was reactive lymphocytes. The IgH gene rearrangement test showed polyclonal B-cell response.

The PCR tests for HSV-1, HSV-2, VZV, TB and toxoplasma for the vitreous fluid were negative as well.

Following the Foscarnet and Kenalog intravitreal injections, the OCT test demonstrated resolution of the subretinal fluid and an MCT reduction to $306 \mu \mathrm{m}$. However, the subretinal lesion advanced and, for the first time, crossed the fovea and almost reached the temporal border of the optic disc (fig. $2 \mathrm{~b}$ ). Therefore, we concluded that there is a progression of the disease under a wide spectrum of antiviral and steroidal treatment. Although no imagining or cytological evidence of PVRL was established, our clinical impression was that PVRL could not be excluded. According to this new working diagnosis, we started empirical treatment with serial intravitreal injections of $400 \mu \mathrm{g}$ methotrexate $/ 0.1 \mathrm{ml}$ twice weekly for 4 weeks, once weekly for 8 weeks and once monthly for 9 months. In addition, the patient was referred to hematology evaluation and underwent positron emission tomography-computed tomography (PET-CT). His systemic examination and PET-CT results were normal.

After 12 injections, the VA was stable and the lesion started to absorb (fig. 1c). In light of the improvement, intravitreal methotrexate treatment and frequent follow-up was continued. Three months later, the patient arrived at the general emergency room with numbness around the right eye and forehead. On examination, a decreased sensation in his right forehead was found. Otherwise, the examination was normal. He underwent a second MRI scan. This time, the MRI scans demonstrated several new space-occupying lesions in the right cerebellum (fig. 3b). Brain biopsy confirmed the diagnosis of CNS lymphoma (fig. 1e, f).

Today, the patient is treated with chemotherapy and radiotherapy. He has no ocular symptoms, his VA did not change, and the lesion in the right eye is absorbed.

\section{Discussion}

The clinical diagnosis of PVRL is challenging due to its tendency to mimic posterior uveitis [8]. The gold standard for diagnosis is biopsy from the vitreous or retina [6]. However, in only $40 \%$ of the patients, the initial diagnosis of intraocular lymphoma is established with a single biopsy [9]. Therefore, the clinical presentation and progression is important for the diagnosis.

In our case, the first AC Tap was positive for VZV. However, the second AC Tap was negative. After having had a comprehensive discussion with the infectious diseases specialists in our institution, we believe it was a false positive result, which is a rare event but could happen in any exam and in any hospital. The cytopathologic diagnosis of the vitreous fluid was standardly fixated and read as reactive lymphocytes. We speculate that the vitritis was reactive and the malignant cell load was retinal and subretinal. Therefore, our vitrectomy examination yielded a false negative result.

The IL-10:IL-6 ratio is an additional laboratory test used for the diagnosis of PVRL, yet its use is controversial because the ratio can raise in neoplastic vitritis but also in inflammatory vitritis [10]. An IL-10:IL-6 ratio greater than 1.0 in suspected cases of PVRL was associated with a sensitivity and specificity of 74.3 and $75.0 \%$, respectively [11]. Cassoux et al. [11] found in 51 vitrectomy specimens from patients with proven PVRL that an IL-10 cut-off value of $400 \mathrm{pg} / \mathrm{ml}$ was associated with $80 \%$ sensitivity and $99 \%$ specificity. Wolf et al. [12] found the ratio was $74.7 \%$ accurate with a sensitivity of $74 \%$ and a specificity of $75.0 \%$. Therefore, although this examination can assist in the diagnosis, since the accuracy of the exam depends on the cut-off level, there is a nonnegligible chance for false positive or nega- 
Zloto et al.: Primary Vitreoretinal Lymphoma Masquerading as Refractory Retinitis

tive answers, especially in lower levels. In our institution, this test is not available. However, we believe that in our case, the test results probably would not change the patient's diagnosis or treatment.

The prognosis of the patients with the disease is poor. 42-92\% of PVRL patients will develop primary CNS lymphoma within a mean of 8-29 months [7]. Recently, Hashida et al. [13] reported that prophylactic systemic chemotherapy for PVRL patients significantly prolonged the time to cerebral involvement. This finding was irrelevant in our case because there was no absolute diagnostic proof of PVRL.

In summary, we describe a case of a patient whose working diagnosis for his disease was PVRL, although there was no initial histopathological evidence to support this diagnosis. The patient was treated with intravitreal injections of methotrexate, and his ocular findings resolved. Five months after the initiation of intravitreal methotrexate treatment, he was diagnosed with CNS lymphoma.

This case should raise the awareness for the importance of the clinical presentation, early diagnosis and treatment of PVRL. Histopathological diagnosis of PVRL should be pursued in every case, yet if there is high clinical suspicion of the disease, a thorough search and intensive follow-up should be planned, and in some cases, empiric chemotherapy treatment can be considered. Early detection of the disease can save vision and prolong the time to cerebral involvement.

\section{Statement of Ethics}

The authors have no ethical conflicts to disclose.

\section{Disclosure Statement}

The authors have no potential conflicts of interest.

\section{References}

1 Coupland SE, Chan CC, Smith J: Pathophysiology of retinal lymphoma. Ocul Immunol Inflamm 2009;17:227237.

-2 Hoffman PM, McKelvie P, Hall AJ, et al: Intraocular lymphoma: a series of 14 patients with clinicopathological features and treatment outcomes. Eye (Lond) 2003;17:513-521.

-3 Akpek EK, Ahmed I, Hochberg FH, et al: Intraocular-central nervous system lymphoma: clinical features, diagnosis, and outcomes. Ophthalmology 1999;106:1805-1810.

4 Levy-Clarke GA, Chan CC, Nussenblatt RB: Diagnosis and management of primary intraocular lymphoma. Hematol Oncol Clin North Am 2005;19:739-749, viii.

-5 Char DH, Kemlitz AE, Miller T: Intraocular biopsy. Ophthalmol Clin North Am 2005;18:177-185, x.

6 Chan CC, Sen HN: Current concepts in diagnosing and managing primary vitreoretinal (intraocular) lymphoma. Discov Med 2013;15:93-100.

7 Sagoo MS, Mehta H, Swampillai AJ, et al: Primary intraocular lymphoma. Surv Ophthalmol 2014;59:503516.

-8 Chan CC, Rubenstein JL, Coupland SE, et al: Primary vitreoretinal lymphoma: a report from an International Primary Central Nervous System Lymphoma Collaborative Group symposium. Oncologist 2011;16:15891599.

-9 Lobo A, Lightman S: Vitreous aspiration needle tap in the diagnosis of intraocular inflammation. Ophthalmology 2003;110:595-599.

10 Akpek EK, Foster CS: Primary intraocular lymphoma with a low interleukin 10 to interleukin 6 ratio and heterogeneous IgH gene arrangement. Arch Ophthalmol 2000;118:731-732. 
Case Reports in

Ophthalmology

\begin{tabular}{l|l}
\hline Case Rep Ophthalmol 2015;6:345-350 \\
\hline DOI: 10.1159/000440762 & $\begin{array}{l}\text { ○ 2015 The Author(s). Published by S. Karger AG, Basel } \\
\text { www.karger.com/cop }\end{array}$ \\
\hline
\end{tabular}

Zloto et al.: Primary Vitreoretinal Lymphoma Masquerading as Refractory Retinitis

11 Cassoux N, Giron A, Bodaghi B, et al: IL-10 measurement in aqueous humor for screening patients with suspicion of primary intraocular lymphoma. Invest Ophthalmol Vis Sci 2007;48:3253-3259.

Wolf LA, Reed GF, Buggage RR, et al: Vitreous cytokine levels. Ophthalmology 2003;110:1671-1672.

13 Hashida N, Nakai K, Saitoh N, et al: Association between ocular findings and preventive therapy with onset of central nervous system involvement in patients with primary vitreoretinal lymphoma. Graefes Arch Clin Exp Ophthalmol 2014;252:687-693.
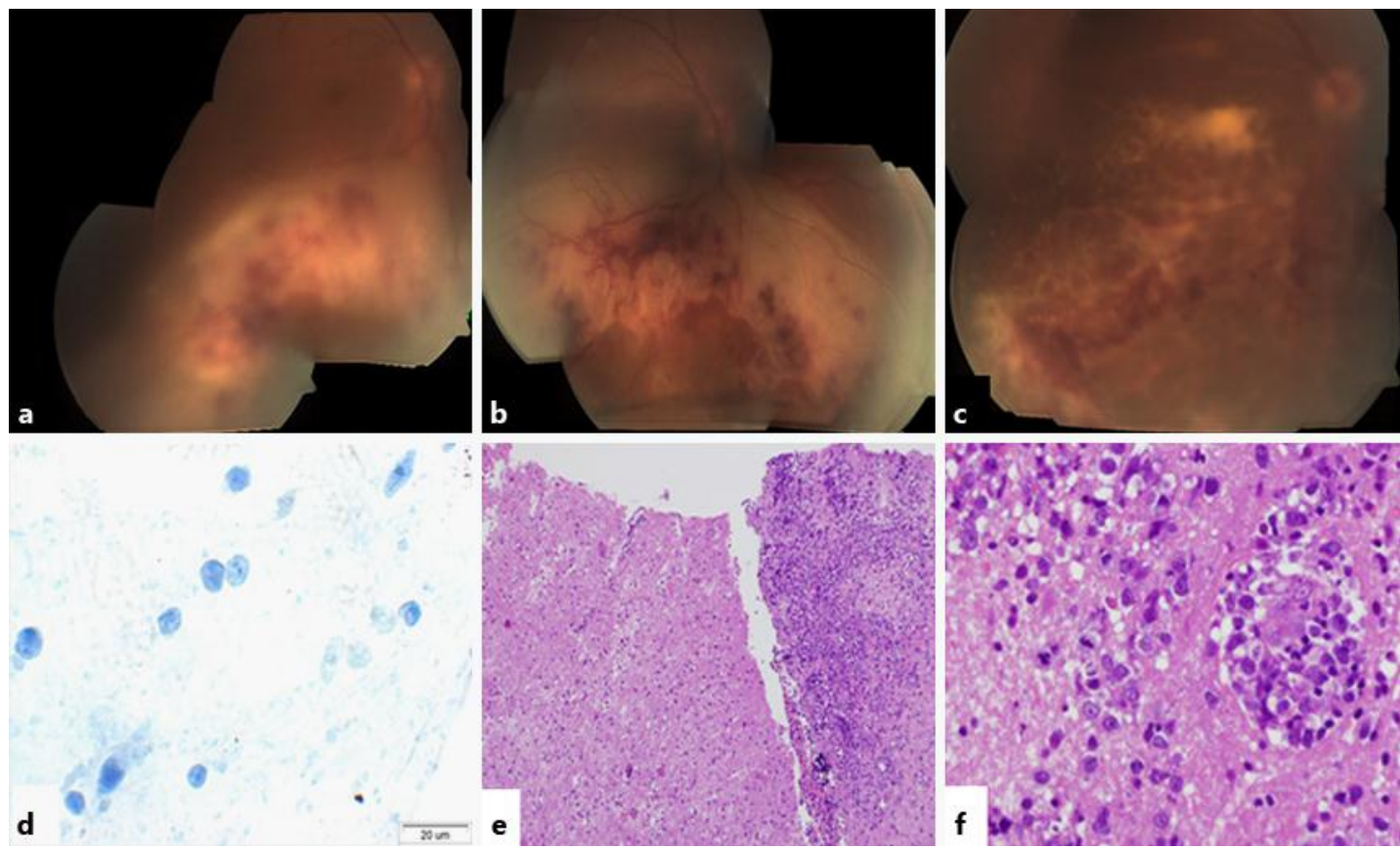

Fig. 1. Posterior segment presentation and histopathological examination. a Vitritis and a white-yellow lesion with few retinal and subretinal hemorrhages in the inferior retina, without macular or optic disc involvement. $\mathbf{b}$ The inferior lesion advanced to the inferior border of the optic disc and involved the macula but not the fovea. $\mathbf{c}$ The subretinal lesion crossed the fovea and advanced to the temporal border of the optic disc. d CD3 staining showed strongly immunoreactive cells. e Brain biopsy. f CNS lymphoma: large lymphocytes with giant nucleus, open chromatin and few mitoses.
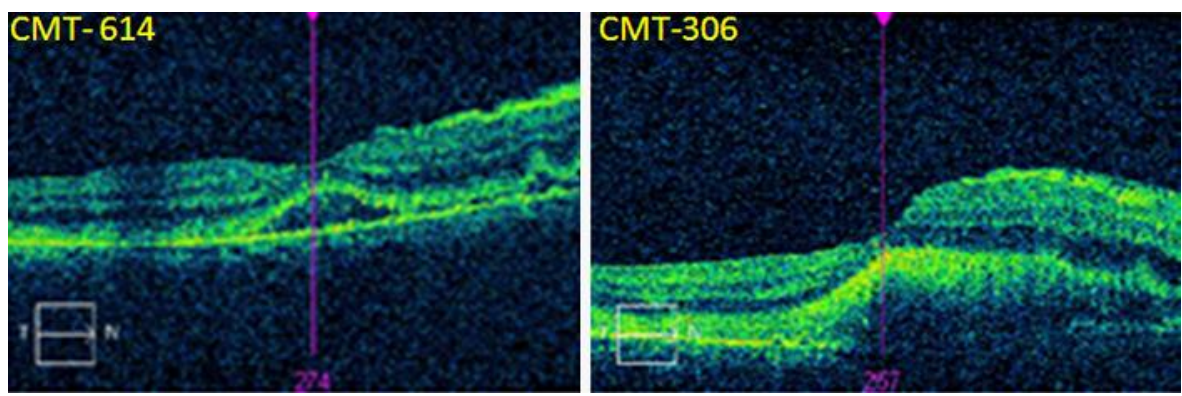

Fig. 2. OCT of the horizontal foveal area. a Presence of subretinal fluid after intravenous acyclovir and oral prednisone treatment. MCT was measured $614 \mu \mathrm{m}$. b Resolution of the subretinal fluid and MCT reduction to $306 \mu \mathrm{m}$ after intravitreal Foscarnet and Kenalog injections. c The subretinal lesion crossed the fovea and farther advanced to the temporal border of the optic disc. 
Case Reports in

Ophthalmology
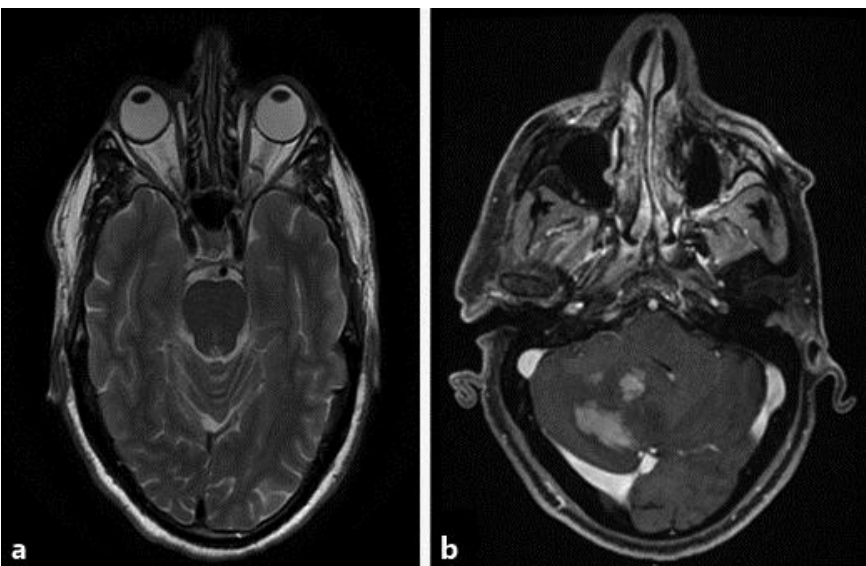

Fig. 3. MRI. a T2 axial cross-section was normal. b T2 axial cross-section showing several new spaceoccupying lesions in the right cerebellum.

Zloto et al.: Primary Vitreoretinal Lymphoma Masquerading as Refractory Retinitis 